\title{
Neumonía neumocócica bacterémica en 45 adultos inmunocompetentes hospitalizados. Cuadro clínico y factores pronósticos
}

\author{
María Luisa Rioseco Z, Raúl Riquelme 0 \\ Bacteremic pneumococcal \\ pneumonia in 45 hospitalized adults
}

Background: The presence of bacteremia during a pneumococcal pneumonia is a sign of bad prognosis. Aim: To report a clinical experience with bacteremic pneumococcal pneumonia. Patients and methods: We reviewed the clinical and laboratory data from 45 adults (36 male, aged 17 to 97 years) with community acquired pneumonia (CAP) and Streptococcus pneumoniae bacteremia, hospitalized between January 1997 and August 2002 at the Puerto Montt Hospital (Southern Chile). Results: Eighty four percent of patients had underlying aggravating conditions, mainly alcoholism (40\%), chronic obstructive lung disease (17.8\%) and renal failure (17.8\%). Seven percent were homeless. Fever, cough, dyspnea and sputum were the most common presenting symptoms. Five patients had pleural involvement. Four strains (8.9\%) of S. pneumoniae had diminished susceptibility to penicillin. Nine patients died (case-fatality rate of $20 \%$ ), but mortality was attributed to pneumonia in only three of them. Main factors associated with a higher mortality were renal failure, absence of cough, an arterial $\mathrm{pH}<7.3$ on admission, ICU hospitalization, shock, mechanical ventilation and an APACHE score $>16$. Conclusions: The high death rate of these patients could be explained mainly by underlying conditions. ICU management and higher cost preventive measures could reduce this rate (Rev Méd Chile 2004; 132: 588-94).

(Key Words: APACHE; Pneumonia, bacterial; Streptococcus pneumoniae)

Recibido el 31 de enero, 2003. Aceptado en versión corregida el 8 de marzo, 2004.

Laboratorio de Microbiología, Servicio de Medicina, Hospital de Puerto Montt.

Streptococcus pneumoniae es el principal agenSe patógeno de las infecciones del tracto respiratorio de niños y adultos, provocando cuadros leves como otitis, sinusitis, etc. y cuadros severos de alta letalidad como lo es la neumonía adquirida en la comunidad (NAC). En aquellos

Correspondencia a: Dra. María Luisa Rioseco Z. Seminario s/n, Puerto Montt. Teléfono: (65) 261166. Fax: (65) 261450.

E-mail: mlrioseco@llanchipal.cl casos de NAC con etiología conocida es el principal agente causal ${ }^{1-4}$.

La letalidad inicial de la NAC neumocócica bacterémica, cercana al 85\%, presentó un importante descenso con el uso de suero hiperinmune y, luego con la aparición de las sulfas y penicilina, llegando a tasas de alrededor de $20 \%$, las cuales no se han reducido significativamente desde la década 1960-695. 
La aparición de cepas de S. pneumoniae resistentes a la penicilina es un problema creciente en el mundo, llegando, en algunos países, a cifras de resistencia global mayores a 30\%3,6-8. Existe controversia acerca de la real relevancia clínica de la resistencia in vitro en ausencia de meningitis y sobre la necesidad de nuevos enfrentamientos terapéuticos basados en los niveles de resistencia ${ }^{9}$. En Chile no existe aún un sistema de vigilancia nacional de sensibilidad y la información disponible, que incluye una mayor proporción de cepas aisladas en niños, muestra niveles de resistencia a penicilina de 10 a $20 \% 7,10,11$. No encontramos comunicaciones que señalen la existencia de cepas con concentración inhibitoria mínima (CIM) $\geq 4 \mathrm{mcg} / \mathrm{mL}$, nivel en que la resistencia se ha asociado con mayor mortalidad en pacientes con NAC bacterémica ${ }^{9}$.

Aproximadamente 25\% de las NAC neumocócicas cursa con bacteremia y constituye un grupo de letalidad significativamente mayor ${ }^{1,12}$ cuya caracterización permitiría un manejo más agresivo que podría mejorar el pronóstico.

En nuestro país hemos encontrado poca información sobre esta particular forma de NAC, siendo el objetivo de este estudio la descripción de algunos de los aspectos clínicos y epidemiológicos de esta entidad, en un grupo de 45 adultos inmunocompetentes manejados en un hospital del sur de Chile.

\section{PACIENTES Y MÉTODO}

El estudio se realizó en el Hospital de Puerto Montt (hospital general de 400 camas). Se analizaron los datos clínico-epidemiológicos de 45 adultos inmunocompetentes, mayores de 15 años, con NAC y hemocultivos positivos a S. pneumoniae hospitalizados entre enero de 1997 y agosto de 2002.

Se consideraron sólo los pacientes que cumplían con los siguientes criterios clínicos ${ }^{13}$ : presencia de un infiltrado nuevo y persistente en la radiografía de tórax al momento de la admisión y presencia de uno o más criterios mayores: a) tos, b) expectoración mucopurulenta o hemoptoica, c) temperatura axilar $>37,8^{\circ} \mathrm{C}$; o por lo menos dos criterios menores: a) dolor torácico pleurítico, b) disnea, c) compromiso de conciencia, d) síndrome de condensación en el examen físico pulmonar, e) leucocitos $>12.000 / \mathrm{mm}^{3}$. Se excluyeron pacientes con antecedentes de hospitalización en los 30 días previos, infección por VIH-1, neoplasia activa, neutropenia (recuento de leucocitos $<1.000 / \mathrm{mm}^{3}$ ), tratamiento quimioterápico o inmunosupresor en los últimos 6 meses o tratamiento con prednisona $\geq 20 \mathrm{mg} /$ día.

La identificación de S. pneumoniae se realizó mediante análisis morfológico de la colonia y test de optoquina. A todas las cepas se les realizó estudio de susceptibilidad a penicilina mediante un antibiograma en difusión con disco de oxacilina. A las cepas resistentes se les determinó la CIM para penicilina y ceftriaxona mediante epsilometría (E test). Para los estudios de susceptibilidad e interpretación de los resultados, se siguieron las recomendaciones del National Committee for Clinical Laboratory Standards ${ }^{14}$.

En todos los pacientes se registraron los siguientes antecedentes clínicos: presencia de comorbilidad, (cardiopatía, diabetes mellitus (DM), enfermedad pulmonar obstructiva crónica (EPOC), asma, insuficiencia renal crónica (IRC), cirrosis u otra enfermedad hepática, cáncer, bronquiectasias o secuelas de tuberculosis (TBC), o alguna otra), hábito tabáquico, alcoholismo, uso de antibióticos (últimos 15 días), lugar de procedencia, sospecha de aspiración, compromiso de conciencia, características del cuadro clínico (fiebre, tos, expectoración purulenta o hemoptoica, dolor torácico, disnea, confusión y escalofríos). Se registró el lugar de hospitalización (sala general, intermedio, unidad de cuidados intensivos (UCI)), signos vitales y resultados de los exámenes de laboratorio al ingreso (recuento de leucocitos, gases arteriales, pruebas de función renal, electrolitos en plasma).

A todos se les tomó una radiografía de tórax al ingreso. El patrón radiográfico fue clasificado en: a) imagen de relleno alveolar, b) intersticial o c) mixto. La extensión del compromiso radiográfico se clasificó en uni o multilobar, uni o bilateral y se registró la presencia de derrame pleural y cavitación. Durante la evolución, se consignó la aparición de cavitación, derrame pleural complicado o empiema y si hubo aumento de las imágenes en $50 \%$ en las primeras $72 \mathrm{~h}$ de hospitalización. 
Se registró el esquema de tratamiento antimicrobiano al ingreso, sus modificaciones y motivo de ellas. También, si existió evidencia de infección extrapulmonar.

Al ingreso, a cada paciente se le calculó la relación entre la $\mathrm{PaO}_{2}$ y la fracción inspirada de oxígeno (PAFI). En todos los pacientes se registró el score de gravedad APACHE II $^{15}$; en aquellos que ingresaron a UCI, se calculó en ese momento y en el resto, utilizando los peores parámetros clínicos y de laboratorio de la evolución.

Se consignó la presencia de shock, insuficiencia renal aguda (IRA), necesidad de UCI, uso de ventilación mecánica (VM), la mortalidad y si ésta fue atribuida a la NAC.

Shock fue definido por presencia de hipotensión arterial (PAS $<90 \mathrm{mmHg}$ ) refractaria al aporte de fluidos o que requiere el uso de agentes vasopresores, o anormalidades de la perfusión tisular evidenciadas por un estado mental alterado, oliguria o acidosis láctica. IRA fue definida por valores de creatinina plasmática inicial 0 en el transcurso de la enfermedad $\geq 2 \mathrm{mg} / \mathrm{dL}$ o aumento $\geq 2 \mathrm{mg} / \mathrm{dL}$ en aquellos pacientes con IRC.

Estadística. Las variables obtenidas fueron almacenadas y analizadas en el programa estadístico SPSS. Para la comparación de las variables paramétricas se usó la prueba t-test de Student y para los datos discretos la prueba Chi-cuadrado y el test exacto de Fisher cuando fue necesario. Un valor de $\mathrm{p}<0,05$ se consideró estadísticamente significativo.

\section{ReSUlTADOS}

En el período de estudio se hospitalizaron 45 pacientes que cumplían con los criterios de inclusión. Las principales características del grupo de pacientes se muestran en la Tabla 1. La edad media fue de 52 años con un rango de 17 a 97 años. El 84\% (38/45) provenía de su hogar, cuatro pacientes fueron trasladados desde otros hospitales y tres correspondían a casos sociales, es decir, sin vivienda conocida. Sólo siete pacientes (16\%) eran previamente sanos y el resto presentaba una o más patologías asociadas (Tabla 1). Ninguno había recibido antimicrobianos en los 15 días previos al ingreso.
Se consignó el hábito tabáquico en 23 pacientes siendo $74 \%$ de ellos no fumador.

En las Tablas 2 y 3 se describen las características clínicas y los hallazgos de laboratorio en la admisión al hospital.

El patrón radiológico fue alveolar en 96\% (43/ 45), unilobar en $67 \%$ (30/45) y multilobar en 33\% (15/45). Los infiltrados pulmonares fueron bilaterales en $22 \%$ (10/45). Al ingreso hubo cavitación

\section{Tabla 1. C aracterísticas clínicas N AC neumocócica bacterémica $(n=45)$}

\begin{tabular}{|lrc|}
\hline & $\mathrm{n}$ & $\%$ \\
\hline Anciano (>65 años) & 14 & 31 \\
Hombres & 36 & 80 \\
Casos sociales & 3 & 6,7 \\
Comorbilidad & 38 & 84 \\
Alcoholismo & 18 & 40 \\
Hepatopatía crónica & 9 & 20 \\
EPOC/asma & 8 & 17,8 \\
Insuficiencia renal crónica & 8 & 17,8 \\
Diabetes mellitus & 7 & 15,6 \\
Secuela TBC & 3 & 6,7 \\
Cardiopatía & 3 & 6,7 \\
Hemodiálisis crónica & 3 & 6,7 \\
Otra comorbilidad & 12 & 26,7 \\
Prednisona (<20 mg) & 4 & 8,9 \\
\hline
\end{tabular}

Tabla 2. Presentación clínica en 45 pacientes con N AC neumocócica bacterémica

\begin{tabular}{|lrc|}
\hline & $\mathrm{n}$ & $\%$ \\
\hline Fiebre & 37 & 82,2 \\
Tos & 37 & 82,2 \\
Disnea & 34 & 75,6 \\
Desgarro & 29 & 64,4 \\
Dolor torácico & 19 & 42,2 \\
Compromiso conciencia & 14 & 31,1 \\
Escalofrío & 7 & 15,6 \\
Sospecha aspiración & 12 & 26,7 \\
Frec. respiratoria $\geq 30 / \mathrm{min}$ & 17 & 44,7 \\
Pa sistólica $\leq 100 \mathrm{mmHg}$ & 13 & 28,9 \\
Frec. cardiaca $\geq 100 / \mathrm{min}$ & 32 & 71,1 \\
\hline
\end{tabular}


Tabla 3. Exámenes de laboratorio al ingreso en pacientes con N AC neumocócica bacterémica

\begin{tabular}{|c|c|c|}
\hline & $\mathrm{n}$ & $\%$ \\
\hline PAFI $\leq 300$ & $21 / 38$ & 55,2 \\
\hline $\mathrm{PaO}_{2} \leq 60 \mathrm{mmHg}$ & $14 / 41$ & 34,1 \\
\hline Hemoglobina $\leq 12 \mathrm{~g} / \mathrm{dL}$ & $21 / 42$ & 50,0 \\
\hline Leucocitos $\geq 20.000 \mathrm{x} \mathrm{mm}^{3}$ & $9 / 43$ & 20,9 \\
\hline Leucocitos $\leq 4.000 \mathrm{x} \mathrm{mm}^{3}$ & $7 / 43$ & 16,3 \\
\hline Baciliformes $\geq 6 \%$ & $10 / 17$ & 58,8 \\
\hline Plaquetas $\leq 100000 \times \mathrm{xm}^{3}$ & $11 / 42$ & 26,2 \\
\hline Sodio $\leq 130 \mathrm{mEq} / \mathrm{L}$ & $6 / 42$ & 14,3 \\
\hline Creatinina $\geq 2 \mathrm{mg} / \mathrm{dL}$ & $10 / 42$ & 23,8 \\
\hline Albúmina $\leq 3,5 \mathrm{~g} / \mathrm{dL}$ & $11 / 23$ & 47,8 \\
\hline APACHE $\geq 16$ & $21 / 42$ & 50,0 \\
\hline
\end{tabular}

En el denominador aparece el número de pacientes con la variable registrada.

en 3 casos (7\%) y derrame pleural en 3 casos (7\%). Durante la evolución hubo derrame pleural complicado en 5 casos (11\%). En tres pacientes $(6,7 \%)$ se detectó progresión de las imágenes radiológicas $>50 \%$ en las primeras $72 \mathrm{~h}$.

Hubo evidencia de infección extrapulmonar en dos casos (4\%) que correspondieron a una otitis media aguda y una meningitis.

Los esquemas antibióticos más frecuentemente usados fueron ceftriaxona (17/45), penicilina sódica asociado a cloranfenicol (14/45) y ceftriaxona más eritromicina (6/45). El esquema inicial fue modificado en $31 \%$ de los casos $(14 / 45)$ y esto ocurrió principalmente al determinar la etiología (8/14). En estos 8 casos se mantuvo como droga única la ceftriaxona o penicilina o se cambió a terapia oral con amoxicilina. Otras causas de modificación fueron deterioro clínico en 2 casos, efectos adversos y mejoría clínica (un caso de cada uno).

En 4 casos (8,9\%) la cepa de S pneumoniae mostró susceptibilidad disminuida a la penicilina (resistente a oxacilina). En tres de estas cepas se determinó la CIM a penicilina, resultando dos $(4,4 \%)$ de susceptibilidad intermedia (CIM 0,12-1 $\mathrm{mcg} / \mathrm{mL})$ y una $(2,2 \%)$ de alta resistencia con $\mathrm{CIM}=2 \mathrm{mcg} / \mathrm{mL}$. Las tres cepas fueron sensibles a ceftriaxona por CIM.

El 13\% (6/45) de los casos requirió hospitalización en UCI. Cinco pacientes recibieron VM (11\%). Seis casos (13\%) presentaron shock durante la evolución. La hospitalización varió entre $1 \mathrm{y}$ 75 días (media \pm DS: $14,5 \pm 15,5$ días) y fue significativamente mayor en los pacientes con antecedente de alcoholismo (media + DS: $17,7+16,5 ; p=0,047)$.

La mortalidad total fue de $20 \%$ (9/45), pero ésta sólo pudo ser atribuida a la neumonía en un tercio del total (3/9). Todos los pacientes que fallecieron eran portadores de una 0 más comorbilidades, siendo las más frecuentes IRC (4 casos, 2 en hemodiálisis crónica), alcoholismo (4 casos) y daño hepático crónico (3 casos). La causa última de fallecimiento fue insuficiencia respiratoria aguda en 5 casos, shock séptico en uno y en el resto no pudo ser determinada. Los factores asociados significativamente a mortalidad obtenidos mediante análisis univariado se muestran en la Tabla 4. No se logró demostrar una asociación significativa de la mortalidad con las siguientes variables: edad $>65$ años y

Tabla 4. Factores pronósticos asociados a mayor mortalidad en N AC neumocócica bacterémica

\begin{tabular}{|lccrrr|}
\hline & $\begin{array}{c}\text { Vivos }(\mathrm{n}=36) \\
\%\end{array}$ & $\begin{array}{c}\text { Fallecidos }(\mathrm{n}=9) \\
\%\end{array}$ & $\mathrm{p}$ & OR & IC 95 \\
\hline Ausencia de tos & 11,1 & 55,6 & 0,03 & 6,4 & $1,2-34,2$ \\
IRC & 11,1 & 44,4 & 0,03 & 6,4 & $1,2-34,2$ \\
Shock & 5,6 & 44,4 & 0,01 & 13,6 & $1,9-94,6$ \\
V mecánica & 5,6 & 33,3 & 0,04 & 8,5 & $1,2-62,1$ \\
Apache $\geq 16$ & 39,4 & 88,9 & 0,02 & 12,3 & $1,4-110,3$ \\
PH $\leq 7,3$ & 9,4 & 44,4 & 0,03 & 7,7 & $1,3-45,5$ \\
Ingreso a UCI & 5,6 & 44,4 & 0,01 & 13,6 & $1,9-94,6$ \\
\hline
\end{tabular}

IRC: Insuficiencia renal crónica. 
$>80$ años, sexo, lugar de procedencia, cardiopatía, DM, EPOC o asma, alcoholismo, daño hepático crónico, hemodiálisis, esteroides previos, secuelas de TBC o bronquiectasias, tabaquismo, dolor torácico, disnea, FR $>30$ por min, fiebre, compromiso de conciencia, sospecha de aspiración, escalofríos, tipo de compromiso radiológico, cavitación, derrame pleural, empiema, infiltrados uni o bilaterales, aumento de los infiltrados en $50 \%$ en $72 \mathrm{~h}$, IRA, infección extrapulmonar, cambio de antibiótico durante la hospitalización, resistencia a la penicilina, PAFI $<300$ y $<200$, albúmina $<3,5$ y $<3$ g/dL, leucocitos $<4.000 / \mathrm{mm}^{3}$ y $>20.000 / \mathrm{mm}^{3}$, plaquetas $<100.000 / \mathrm{mm}^{3}$ y número (2 ó 3) de comorbilidades.

\section{Discusión}

El principal hallazgo de nuestro estudio fue definir un subgrupo de pacientes adultos hospitalizados por NAC con alta mortalidad $(20 \%)$ y frecuente morbilidad asociada (84\%). El 8,9\% de las cepas de S. pneumoniae presentó susceptibilidad disminuida a penicilina. La mortalidad se asoció en forma significativa con ausencia de tos, IRC, shock, $\mathrm{pH}$ al ingreso $\leq 7,3$, necesidad de $\mathrm{VM}$, APACHE $\geq 16$ e ingreso a UCI.

En nuestra casuística, la mortalidad global fue de $20 \%$, pero en sólo 3 de 9 pacientes la causa de muerte pudo ser atribuida directamente a la NAC, debido a que en los seis casos restantes, la morbilidad asociada fue el factor determinante. La alta prevalencia de morbilidad asociada en los pacientes que presentan una neumonía neumocócica bacterémica, hecho frecuentemente descrito en la literatura, podría explicar la persistencia de altas tasas de letalidad en este particular tipo de NAC, a pesar del uso de antimicrobianos específi$\cos ^{16-18}$. En un estudio de 46 casos de NAC neumocócica en Santiago, donde se incluyeron 24 pacientes bacterémicos, también se observó elevada comorbilidad (82\%) pero con una letalidad inferior (12,5\%) a la encontrada por nosotros. Este mejor pronóstico podría explicarse por una menor prevalencia de alcoholismo (14\% vs $40 \%$ ) y a un manejo más agresivo, reflejado en una mayor frecuencia de ingreso a UCI (39\% vs $13 \%)^{19}$.

El alcoholismo es un factor conocido de mal pronóstico de las NAC neumocócicas bacterémi- cas $^{12}$, pero nosotros, a pesar de la alta prevalencia de pacientes alcohólicos, no logramos demostrar una asociación con mayor mortalidad, probablemente por lo reducido de nuestra casuística. Lo mismo es válido para los pacientes de edad avanzada ${ }^{3,5,12,18}$. El alcoholismo si impactó significativamente en la duración de la hospitalización, revelando una evolución clínica más complicada y una respuesta más lenta al tratamiento.

Se ha señalado un aumento en la incidencia de bacteremia por S. pneumoniae, lo que ha sido atribuido al envejecimiento de la población y a una mayor prevalencia de enfermedades predisponentes a infecciones invasivas por este agente y de infectados por VIH-13,20. En este estudio, la primera condición no está presente ya que se trata de una población relativamente joven (edad promedio 52 años), en que sólo un tercio superaba los 65 años y no se incluyó ningún paciente infectado por VIH-1. Sin embargo, coincidimos en la alta prevalencia de morbilidad asociada (84\%) destacando el alcoholismo, la enfermedad crónica del hígado, la IRC y la EPOC o asma. Nosotros también hemos observado en los últimos años un aumento en el aislamiento de S. pneumoniae en sangre en pacientes adultos, pero esto es coincidente con un mayor número de hemocultivos debido a la aplicación de normas nacionales para el manejo de esta patología que incluyen la recolección de este examen ${ }^{21}$ y a la realización de un estudio prospectivo de etiología de NAC en adultos.

La mayoría de los pacientes analizados presentaba fiebre, tos y disnea; el dolor pleurítico, generalmente asociado a neumonía neumocócica, sólo estaba presente en el $42 \%$ de los casos. La ausencia de fiebre, hecho que traduciría una deficiente función granulocitaria, ha sido señalado como un factor asociado a mayor mortalidad ${ }^{18,22}$. En esta casuística, el 16\% (6/37) de los pacientes con fiebre al ingreso falleció versus el 37\% (3/8) de los pacientes afebriles, pero esta diferencia no alcanzó significación estadística $(\mathrm{p}=0,326)$.

No se pudo demostrar que la mayor extensión del compromiso radiológico de la NAC se asociara a mal pronóstico; sin embargo, hubo clara correlación con otros factores que traducen mayor gravedad como son acidosis, shock, ingreso a UCI, uso de VM y APACHE $\geq 16$. 
La elección de ceftriaxona (sola o asociada) como terapia empírica en más de la mitad de los casos, se correlaciona con la permanente aparición de reportes nacionales y mundiales que advierten del rápido incremento de la resistencia de S. pneumoniae a penicilina, antimicrobiano que hasta hace algunos años era considerado el tratamiento de elección de la neumonía neumocócica $^{8,20,23}$. Considerando las variaciones regionales en los niveles de resistencia, con centros de Santiago que reportan cifras de alrededor de $15 \% 11,19$ y niveles locales significativamente más bajos, además de la ausencia de reportes de cepas con CIM >4 mcg/mL, nivel que ha sido correlacionado con mal pronóstico de NAC, creemos que en la formulación de normas terapéuticas se deben acoger estas diferencias, respaldando el uso de antimicrobianos de menor costo como penicilina 0 amoxicilina en algunas regiones.

\section{REFERENCIAS}

1. Fine M, Smith M, Carson C, Mutha S, Sankey S, WeISSFELD L ET AL. Prognosis and outcome of patients with community-acquired pneumonia: a meta-analysis. JAMA 1996; 275: 134-41.

2. Saldías F, Mardónez J, Marchesse M, Viviani $P$, FARÍAS, Díaz A. Neumonía adquirida en la comunidad en el adulto hospitalizado. Cuadro clínico y factores pronósticos. Rev Méd Chile 2002; 130: 1373-82.

3. OrtQvist A. Pneumococcal disease in Sweden: experiences and current situation. Am J Med 1999; 107 (1 A): 44S-49S.

4. FILE T, TAN J. Incidence, etiologic pathogens and diagnostic testing of community-acquired pneumonia. Curr Op Pulmonary Med 1997; 3: 89-97.

5. WatanaKunaKorn C, Bailey T. Adult bacteremic pneumococcal pneumonia in a community teaching hospital, 1992-1996. Arch Intern Med 1997; 157: 1963-71.

6. Appelbaum PC. Antimicrobial resistance in Streptococcus pneumoniae: an overview. Clin Infect Dis 1992; 15: 77-83.

7. Heitmann I. Epidemiología nacional de las infecciones causadas por Streptococcus pneumoniae. Rev Chil Infect 1999; 16: 133-6.
Identificamos un grupo de pacientes con NAC de características bien definidas, con predominio de sexo masculino (80\%), frecuentemente alcohólicos, portadores de enfermedades crónicas principalmente IRC y daño hepático crónico, con 6,7\% de casos en situación de abandono social y que fallecen en $20 \%$. Considerando que los recursos disponibles para salud son limitados, eventuales medidas preventivas como el uso de la vacunación antineumocócica podrían focalizarse a este tipo de pacientes de mayor riesgo.

A la luz de estos hallazgos y considerando las limitaciones de un estudio retrospectivo, nos parece importante promover la realización de un estudio prospectivo y multicéntrico que permita conocer en forma más fidedigna las características clínicas y epidemiológicas de este tipo de pacientes en nuestro medio.

8. Doern G, Brueggemann A, Huynh H, Wingert E, RHomberg P. Antimicrobial resistance with Streptococcus pneumoniae in the United States, 19971998. Emerg Infect Dis 1999; 5: 757-65.

9. Niederman M, Mandell L, Amzueto A, Bass J, Broughton W, Campbell G et al. Guidelines for the management of adults with community-acquired pneumonia. Diagnosis, assessment of severity, antimicrobial therapy and prevention. Am J Respir Crit Care Med 2001; 163: 1730-54.

10. GonZÁLEZ P. Vigilancia de la resistencia a antimicrobianos. Rev Chil Infect 2002; 19 (Supl.2): S1359.

11. Soler T, Salamanca L, Arbo G, Molina E. Estudio de sensibilidad in vitro de cepas de Streptococcus pneumoniae de infecciones respiratorias bajas en el Instituto Nacional del Tórax. Rev Méd Chile 2002; 130: 304-8.

12. Musher D, Alexandraki I, Graviss E, Yanbeiy N, Eid A, INDERIAS L ET AL. Bacteremic and nonbacteremic pneumococcal pneumonia. Medicine 2000; 79: 210-21.

13. Fang GD, Fine M, Orloff J, Arisumi D, Yu VL, SINGER D ET AL. New and emerging etiologies for community-acquired pneumonia with implications for therapy: A prospective multicenter study of 359 cases. Medicine 1990; 69: 307-16. 
14. National Committee for Clinical Laboratory StanDARDS. Performance standands for antimicrobial susceptibility testing; twelfth informational supplement. 2002; $22 \mathrm{~N}^{\circ} 1$

15. Knaus WA, Draper EA, Wagner DP, Zimmerman JE. APACHE II: A severity of disease classification system. Crit Care Med 1985; 13: 818.

16. Afessa B, Greaves W, Frederick W. Pneumococcal bacteremia in adults: a 14- year experience in an Inner-city University Hospital. Clin Infect Dis 1995; 21: 345-51.

17. Kalin M, Ortqvist A, Almela M, Aufwerber, Dwyer R, Henriques B et al. Prospective study of prognostic factors in community acquired bacteremic pneumococcal disease in 5 countries. J Infect Dis 2000; 182: 840-7.

18. Marfin A, Sporrer J, Moore P, Siefrin A. Risk factors for adverse outcome in persons with pneumococcal pneumonia. Chest 1995; 107: 457-62.
19. Díaz A, Torres $C$, Flores L, García $\mathrm{P}$, Saldías $\mathrm{P}$. Neumonía neumocócica adquirida en la comunidad en adultos hospitalizados. Rev Méd Chile 2003; 131: 505-14.

20. Karstaedt S, Khoosal M, Crewe-Brown H. Pneumococcal bacteremia in adults in Soweto, South Africa, during the course of a decade. Clin Infect Dis 2001; 33: 610-4.

21. SOCIEDAD Chilena de EnFermedades Respiratorias. Consenso Nacional en Neumonías Adquiridas en la Comunidad en Adultos y Niños. Rev Chil Enf Respir 1999; 15: 67-136.

22. Torres J, Cárdenas O, Vásquez A, Schlossberg D. Streptococcus pneumoniae bacteremia in a community hospital. Chest 1998; 113: 387-90.

23. Raz R, Elhanan G, Shimoni Z, Kitzes R, Rudnicki C, IgRA Y ET aL. Pneumococcal bacteremia in hospitalized Israeli adults: epidemiology and resistance to penicillin. Clin Infect Dis 1997; 24: 1164-8. 